\title{
Hedonic integration: Test of a linear model
}

\author{
MICHAEL D. KLITZNER \\ University of California, San Diego, La Jolla, California 92037
}

\begin{abstract}
A simple adding model was tested for the integration of the hedonic component of taste stimuli. Subjects tasted mixtures of quinine sulfate and apple juice and rated the pleasantness of the composite taste. The rating data showed significant nonadditivity, which could reflect failure of the model itself or merely a nonlinear response output function. Since the rating data could be transformed to additivity, justification for such transformation was sought through a second task based on the logic of two-stage integration. In this task, subjects rated the difference between taste mixtures. The subtracting model underlying this judgment provided functional scale values for the taste mixtures on validated interval scales. These functional scale values were consistent with the predictions of the simple adding model, and thus justified the transformation of the single rating data to additivity. The application of functional measurement to obtain the psychophysical functions of the two taste components is also illustrated.
\end{abstract}

This study addresses a problem that has been of interest to psychologists for over 40 years: does a simple additive model underlie the process of hedonic integration". Early work with color and odor combinations (Guilford. 1931; Spence \& Guilford, 19.33) attempted to validate a linear model for hedonic integration by predicting the pleasantness of combinations from ratings of the separate stimuli. While the work of Spence and Guilford is suggestive and of historical interest. the tests of model fit presented were far from conclusive. In fact, few studies have presented convincing, quantitative tests of a model for hedonic integration. Recent developments in functional measurement methodology have provided powerful tools for model analysis. Applying these tools, this study presents a quantitative test of a simple linear model for the integration of the hedonic component of taste stimuli.

The major concern of this paper is stimulus integration: discovery of the model underlying hedonic integration for taste would have broad implications for both the study of taste as a subjective experience and for the study of taste as a psychophysical phenomenon. A secondary concern is the discovery of the function which relates pleasantness to intensity for the individual taste components. This psychophysical law is derived as a byproduct of the more important law of stimulus integration, and pleasantnessintersity functions for the individual taste stimuli employed will be presented.

This work was completed while the author was a National Science Foundation Graduate Fellow and was supported by NSF Grant GS-36918 and facilitated by grants from the National Institute of Mental Health to the Center for Human Information Processing. University of California, San Diego. The author wishes to thank Norman H. Anderson for his invaluable assistance throughout this project. Thanks are also extended to Stanley Parker for running the subjects. Reprint requests should be sent to the author. Department of Psychology. University of California, Lit Jolla. California 92037.
Information integration theory (Anderson, 1974a, b) provides the framework necessary for the development and evaluation of an additive model for hedonic integration. The model tested in this study relates to a general algebraic formulation employed extensively by Anderson and others in the study of human judgment. As applied to the combination of subjective tastes, the model may be written,

$$
R_{i j}=(w s)_{i}+(w s)_{j} \text {. }
$$

$R_{i j}$ is the response to a combination of taste components, and $\mathrm{w}$ and $\mathrm{s}$ are the subjective hedonic parameters of the taste components relative to some dimension of judgment, in this case, "pleasantness." If the model is correct, and if the observed response is on an interval scale, then the $R_{i j}$ must plot as parallel lines when the stimuli are presented in a factorial design (see Anderson, 1974a). This prediction of parallelism thus provides a direct visual test of the additive model.

A preliminary experiment was conducted in which subjects rated the pleasantness of mixtures of various concentrations of quinine sulfate and apple juice. But, instead of conforming to the predictions of the simple adding model (Equation 1), the data showed significant nonparallelism. Unfortunately, nonparallelism is equivocal: it might reflect a nonadditive integration rule, or merely a nonlinearity in the response output function. Thus it could be argued that the nonparallelism merely reflected a nonlinear or ordinal response measure. Indeed, the nonparallelism could be transformed out.

Some writers in measurement theory (e.g., Krantz, Luce. Suppes, \& Tversky, 1971) have taken the position that data that can be transformed to additivity should be so transformed. However, nonparallelism could result if a weighted averaging model applied instead of a simple adding model 
(Anderson, 1974a), and in that case transformation to additivity would not be justified. It seemed necessary, therefore, to adopt the more conservative position that some substantive justification is necessary before a response scale transfornation is applied (Anderson. 1962; Bogartz \& Wackwitz, 1971). For this purpose, an approach based on two-stage integration was adopted (Anderson, 1974b, Section II.B.11).

The two-stage approach was implemented by adding a second condition in which subjects rated the preference between two mixtures. In this condition, there are two integration stages. In the first stage, the components of each mixture are integrated to obtain the pleasantness value for each separate mixture. In the second stage. the difference in preference between these two mixtures is obtained.

The model for this two-stage integration task can be written,

$$
R_{i j k l}=\left[(w s)_{i}+(w s)_{j}\right]-\left[(w s)_{k}+(w s)_{1}\right] .
$$

The addition sign ( + ) within the brackets denotes, in this case, merely a summative operation which may or may not be a simple addition. The preference judgments should obey the simple subtracting model (Equation 2) regardless of whether the component tastes obey an additive integration rule. The subtracting model is easily tested, since it predicts no interactions in the analysis of variance test. If this prediction is verified, then the model yields functional scale values of the separate taste mixtures on validated interval scales. These scale values can then be compared to the single ratings of the taste mixtures. That gives a substantive criterion to determine whether or not to employ a response scale transformation on the single ratings.

\section{METHOD}

Subjects participated in two judgment tasks. The first was a replication of the preliminary experiment (single ratings), and the second was designed to allow a two-stage integration analysis (preference ratings).

\section{Stimuli \\ Five levels of commercially available apple juice $(20 \%, 35 \%$, $50 \% .65 \%$, and $80 \%$ ) were combined factorially with three levels of quinine sulfate solution (Towne, Paulson, \& $\mathrm{Co}$. pharmaceutical grade, in 20,60 , and $100 \mathrm{ppm}$ concentrations) to form a 5 by 3 design. In addition, a high-anchor stimulus was constructed using a $90 \%$ concentration of apple juice, and a low-anchor stimulus was constructed using $140 \mathrm{ppm}$ quinine in distilled water.}

\section{Procedure}

Each subject rated two replications of the 5 by 3 factorial design. After initial instructions were given by the experimenter, the experiment was controlled through instructions displayed by the video Teletype terminal of the PDP-12 computer. Responses were made by moving a marker on a computer-displayed graphic rating scale bearing the legends "VERY GOOD" and "VERY BAD." Before the actual experiment, the subject was given 10 practice trials to familiarize him with the response device. During this practice, the anchor stimuli were introduced. The subject was told to assume that these stimuli uould be somewhat better or somewhat worse than the experimental stimuli. thus defining the range of the rating scale. A standard sip-and-spit method was employed. and subjects rinsed between each trial with distilled water. The anchor stimuli were presented twice each during each replication of the design.

The four corner stimuli from the above 5 by 3 factorial were combined with two middle stinuli $65 \%-20 \mathrm{ppm}, 35 \%-100 \mathrm{ppm}$ of apple juice and quinine. respectively) to form the 4 by 2 design for the preference judgments. In this task. subjects tasted both a row and a column stimulus and rated the preference between them. The rating scale for this task bore the legend "NO DIFFERENCE" in the middle. and markers at either end to indicate the direction of preference. After a practice trial, the subject rated two replications of the 4 by 2 factorial. Again, a sip-and-spit method was employed and the subject rinsed with distilled water after each pair. Due to error, one data point was lost for four subjects and was estimated. Each of the 20 subjects was paid $\$ 1.88$ for his services.

\section{RESULTS}

\section{Hedonic Summation}

Figure la plots the single ratings of the taste mixtures. Although these data appear approximately additive. closer inspection reveals that the curves tend to converge as the concentration of apple juice increases. This deviation from parallelism was statistically significant, as shown by the ANOVA test of interaction, $F(8,152)=3.52$. These data are quite similar to those obtained in the preliminary experiment, which tested 24 subjects in a similar design.

Two interpretations of this deviation from additivity are possible as noted in the introduction. The first. and perhaps the simplest, is that the integration process is additive and that the nonparallelism merely represents nonlinearity in the rating response. On this interpretation, a monotone transformation could be found that would eliminate the nonlinearity. In fact, a simple logarithmic formula was able to render the data parallel as shown in Figure 16 . ANOVA of these transformed data showed a nonsignificant interaction. $F(8,152)=1.11$. As indicated earlier, many investigators would accept these results as support for an additive model.

The second possible interpretation of these results is that the deviations from parallelism reflect a nonlinear integration process. In other areas of human judgment, considerable evidence has been found to support an averaging rule over a simple adding rule (Anderson, 1974a). Although these two models both predict parallelism under certain conditions, the averaging model would predict deviations from parallelism if the weight parameter (w) depended on the concentration value. To apply this model to the data of Figure la would require that the relative weight of apple juice increase as a function of its concentration, or equivalently, that the relative weight of quinine increase as apple juice 


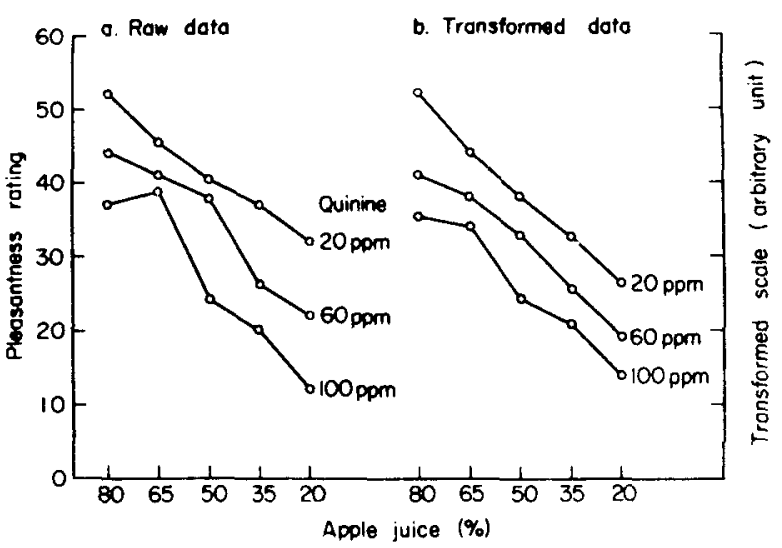

Figure 1. Pleasantness ratings of quinine sulfate-apple juice mixtures. Panel a, raw data; Panel b, log-transformed data.

concentration decreases. This weighted average interpretation seems plausible on the ground that quinine may become more salient in lower concentrations of apple juice.

It seems clear, therefore, that some substantive criterion is needed to justify any transformation to additivity. It was for this purpose that the preference task was included. This task involves two successive stages: First, the integration of quinine and apple juice to yield the hedonic value of each separate taste mixture; second, the judgment of the difference between these two hedonic values. The difference judgments in the second stage should not be affected by any nonadditivity that may be present in the first stage. Accordingly, they should obey a linear, subtracting model regardless of whether or not the first stage is additive.

The mean difference judgments for the 4 by 2 preference design are shown in Table 1 . Since the interaction between row and column factors was not significant, $F(3.57)=1.81$, these data support the subtracting model (Equation 2) for the preference judgments.

The special virtue of this two-stage approach is that the subtracting model provides a criterion for justifying the transformation to additivity of the single taste data of Figure 1. This justification rests on two grounds. The first ground is that the column means in Table 1 are the hedonic values of the single taste stimuli in the four columns. In fact, these hedonic values are on a validated interval scale because of the success of the subtracting model. This validation of a response measure through a test of model fit illustrates a general property of functional measurement (see Anderson, 1974a, Section I.1.5). In the present case, this property follows directly from the linearity of Equation 2.
The second ground is that the four column stimuli in the preference task in Table 1 were chosen to have a particular relationship to the single taste stimuli from the main, 3 by 5 , design. In fact, the four column stimuli in Table 1 are the four corner stimuli from the 3 by 5 design of Figure 1. Thus, the four column stimuli in Table 1 form a 2 by 2 design, as can be seen in the table.

Now, since the column means in Table 1 form a validated interval scale of the hedonic values of the four single taste stimuli, they provide a criterion for determining whether the nonadditivity in Figure 1a is real or not. This criterion is obtained by plotting the four column means in 2 by 2 form. If the nonadditivity in the single taste data is real, it will reappear in this 2 by 2 graph as a similar nonparallelism. Conversely, if this 2 by 2 graph exhibits parallelism, then the nonadditivity in Figure 1a is not real and the transformation to additivity is justified.

This 2 by 2 graph is shown in Figure 2. By visual inspection, the curves appear to conform to the parallelism prediction nicely. The interaction did not approach significance, $F(1,19)=.14$. Thus, an explanation of the nonparallelism in Figure 1 a based on a nonlinear integration process seems to be inappropriate. The data in Figure 2 indicate that the nonparallelism in Figure 1a resulted from a nonlinear response output function for the single rating data. Therefore, the transformation of the single rating data is justified, and Figure $1 \mathrm{~b}$ can be taken to show that the true integration process is one of hedonic summation.

\section{The Psychophysical Law}

The relationship between physical stimulus intensity and pleasantness is derived in the simplest possible way from Figure 1b. This psychophysical law is merely the function relating subjective stimulus scales to the physical stimulus magnitude, in this case, concentration. Subjective hedonic magnitude is precisely what the subjects were asked to judge in the single rating task. Thus, the intensity-pleasantness

Table 1

Preference Ratings

\begin{tabular}{|c|c|c|c|c|}
\hline & $\begin{array}{c}80 \% \\
\text { Juice } \\
100 \text { ppm } \\
\text { Quinine }\end{array}$ & $\begin{array}{c}80 \% \\
\text { Juice } \\
20 \text { ppm } \\
\text { Quinine }\end{array}$ & $\begin{array}{l}20 \% \\
\text { Juice } \\
100 \text { ppm } \\
\text { Quinine }\end{array}$ & $\begin{array}{c}20 \% \\
\text { Juice } \\
20 \mathrm{ppm} \\
\text { Quinine }\end{array}$ \\
\hline $\begin{array}{l}65 \% \text { Juice } \\
20 \text { ppm Quinine }\end{array}$ & 32.0 & 51.0 & 12.0 & 28.0 \\
\hline $\begin{array}{l}35 \% \text { Juice } \\
100 \mathrm{ppm} \text { Quinine }\end{array}$ & 35.0 & 53.0 & 17.0 & 36.0 \\
\hline Means & 33.5 & 52.0 & 14.5 & 32.0 \\
\hline
\end{tabular}




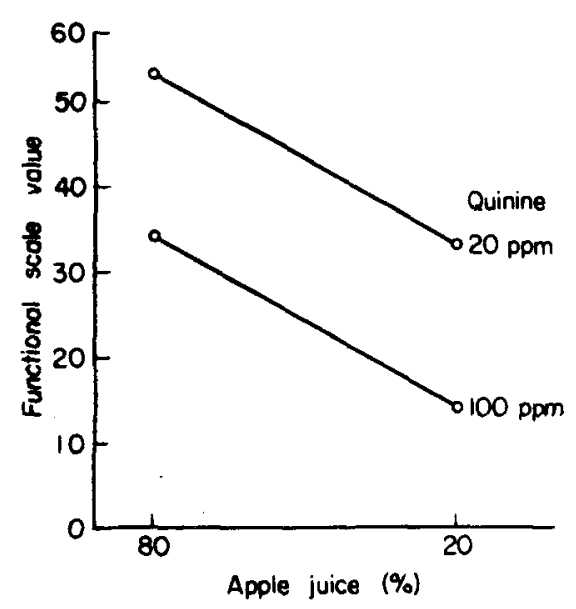

Figure 2. Functional scale values of quinine sulfate-apple juice mixtures derived from the preference task.

function for apple juice may be visualized as the mean of the three curves in Figure 1b. Inspection of Figure $1 b$ reveals that this function has a slight negative acceleration. Such a psychophysical relationship for sweetness is consistent with that reported by Moskowitz (1974), who found a negative acceleration for glucose pleasantness as a function of increasing concentration.

The psychophysical function for quinine may be visuakized in an analogous manner. The separation between the curves in Figure $1 \mathrm{~b}$ represents the subjective hedonic scales for this factor. Inspection of Figure $1 \mathrm{~b}$ reveals that the pleasantness-intensity function for quinine is essentially linear over the range studied.

\section{DISCUSSION}

\section{Hedonic Summation}

The present data provide some support for simple hedonic summation, at least for bitter and sweet taste components. The logic of this support deserves closer consideration. The raw data from the single ratings were actually nonadditive, but they could be transformed to additivity, both in the preliminary experiment and in the experiment reported here. Many investigators would consider that to be adequate support for the simple adding model.

But there is a problem, as noted in Anderson (1962): It is often too easy to transform nonadditive data to additivity, even when the proposed model is wrong. In the present study, there was some reason to expect nonadditivity since a weighted averaging model might apply if quinine became more salient in lower apple juice concentrations. Thus, some further criterion justifying transformation was desirable. The two-stage logic as implemented in the preference task provided an independent test of the integration rule which should not be affected by differential weighting. This two-stage approach supported the adding model. Such support is not conclusive, but it provided a much stronger basis for transforming the single rating data to additivity.

\section{Two-Stage Integration}

These results demonstrate the potential methodological usefulness of the two-stage integration approach. Response scale validity must be of primary concern when testing algebraic models for hedonic integration. The two-stage approach allows a model to be tested directly, as with the single taste mixtures in the present case, and indirectly through a task such as the difference ratings. When data from one stage of the two-stage process allow for two interpretations, values derived from the second stage may be used to determine if it is the proposed model or merely the response output function that is faulty. Such information may provide justification for a response scale transformation or indicate that such a transformation is inappropriate; on the other hand. such information may merely confirm conclusions drawn from the first integration stage.

The present approach is different from the two-stage logic employed by Curtis, Attneave, and Harrington (1968). Their two stages represent stimulus input, in which each physical stimulus is transduced into its subjective value, and response output, in which the internal response is made overt. In contrast, the two stages in the present approach represent two successive stages of stimulus integration that lie between the stimulus input and the response output.

However, there are some interesting similarities between functional measurement and the approach used by Curtis et al. These can be best explained with reference to the functional measurement diagram (e.g., Anderson, 1974b, Figure 12). In terms of this diagram, their input stage corresponds to the psychophysical law that relates physical stimulation to its subjective value. Similarly, their output function corresponds to the psychomotor law which relates the internal response to the response instrument employed by the experimenter. Thus, the present log transformation corresponds to their output function.

The approach of Curtis et al. is also based on using an algebraic model. From the present standpoint, that is really the central feature of their approach because the model provides a framework for obtaining a monotone transformation of the response. This reliance on an algebraic model of stimulus integration is also, of course, the fundamental characteristic of functional measurement.

The present two-stage approach adds a second integration model in order to get a firmer grip on the question of response transformation. The view of Curtis et al. seems to be similar to that of Krantz et al. (1971), namely that data that can be 
transformed to additivity should be so transformed. The present approach is more conservative, since it also asks for consistency of these transformed values with those obtained from the second integration stage. A similar application of the two-stage logic of functional measurement has been given by Birnbaum and Veit (1974). This two-stage approach exemplifies the criterion of converging operations discussed by Garner, Hake, and Eriksen (1956).

\section{Stimulus Interaction}

Bitter compounds such as quinine appear to show minimal cross-adaptation effects (McBurney, Smith, \& Shick, 1972). Thus, an additive model for hedonic integration involving quinine is plausible, although by no means the only candidate. However, many tastes such as sour and salty compounds are known to show cross-adaptation (McBurney et al., 1972). When interacting substances are employed, a simple adding model no longer applies, and the data will be nonparallel or nonlinear. But then some criterion is necessary to distinguish those nonlinearities which reflect the true integration process from those which merely reflect a nonlinear output function.

The two-stage integration logic can be of great use in exploring the hedonic integration of interacting tastes. For example, a study similar to the present experiment might be conducted employing salt and sucrose as stimuli. Since salty tastes do cross-adapt, an additive model is not a candidate for this case of hedonic summation. However, an analysis of variance type model in which each taste has a main effect and there is also a term representing the interaction of the two tastes might reasonably be considered. Theoretical curves generated from the model would provide a test of model fit. However, should the data deviate from the predicted pattern, a problem similar to that caused by the nonparallelism of the single rating data in the present study would arise. It is very difficult with only a single task to differentiate between deviations from the proposed model which are caused by a nonlinear output function and those which may be caused by the model itself being wrong.

However, by employing a two-stage task such as the preference task, subjective values for the taste mixtures may be derived on a validated interval scale. When these values are plotted, they will be similar either to the theoretical curves generated from the model or similar to the data obtained from the single rating task. It the former is the case, then it is the response output function for the single ratings that is faulty, and a transformation is needed. Contrariwise, if the latter is the case, then the proposed model is faulty. and a model that accounts for the observed data must be developed. A comprehensive theory of hedonic integration will need to deal with tastes that interact, an the two-stage integration logic may prove particularly useful in such investigations.

\section{Conceptual Status of the Additive Model}

The hedonic integration model presented here is a conceptual model, and it does not necessarily mirror the physiological integration process. Hedonic integration may rely heavily or even exclusively on receptor rather than cognitive phenomena. Nonetheless, a conceptual model of the sort developed and tested in this paper can be of use in investigations where subjective taste phenomena are of primary interest.

It has been suggested that since intensities summate on the receptor level in some instances, hedonic integration must operate on the output of sensation magnitude. This point is well taken if the present model is to claim a physiological, mechanistic status. However, no such claim is being made. Because the present model is conceptual, hedonic magnitude is itself conceptual; both are theoretical tool to aid in the quantification, prediction, and hence the understanding of human judgment behavior in the area of hedonics.

A similar issue arises in Anderson's (1972) treatment of the size-weight illusion. Anderson conceptualizes the illusion by assuming the presence of a "visual" heaviness cue which is averaged with the subjective heaviness of the judgment object. The psychological reality of a "sensation magnitude" for the visual cue is not of interest. What is of interest is that Anderson's conceptual model can predict the subjects' behavior with a great degree of accuracy. His model, whether physiologically accurate or not, makes a substantive contribution to the understanding of psychophysical judgment.

While the present model does not claim to be physiological, the fact that it seems to describe the subjective likeableness of bitter/sweet mixtures can help direct research in receptor psychophysics. Conceptual and physiological models can interact in a productive fashion to develop an integrated science of human taste perception. Both types of models can make substantive contributions to our understanding of cognition and physiology.

\section{Functional Measurement and the Psychophysical Law}

Some critics of information integration theory have raised the question of whether functional measurement methodologies can yield the psychophysical law. The answer is yes: the present study is a case in point. As illustrated in the results, the relationship between intensity and pleasantness may be derived from the marginal means of a factorial design in which individual tastes are judged in combination. While the psychophysical law was of tangential interest to the present investigation, it is, nonetheless, available through functional measurement.

Functional measurement's ability to deal with both psychophysical issues and with questions of stimulus 
integration may become important in an area closely related to hedonic integration, namely additivity of taste intensities. The present approach of combining two integration tasks in order to validate both the model and the response scale seems a promising one in this area. Such cross-task validation has proved successful in other areas of psychophysical judgment (Anderson, 1972, 1974b). Its application to taste intensity integration seems worthy of further consideration.

\section{REFERENCES}

Anderson. N. H. On the quantification of Miller's contlict theory, Psychological Review, 1962, 69, 400-414.

ANDERSON, N. H. Cross-task validation of functional measurement. Perception \& Psychophysics. 1972. 12, 389-395.

ANDERSON, N. H. Information integration theory: A brief survey. In D. H. Krantz. R. C. Atkinson. R. D. Luce, and P. Suppes (Eds.). Contemporany developments in mathematical psychology (Vol. 2). San Francisco: Freeman, 1974. (a)

Anderson, N. H. Algebraic models in perception. In E. C. Carterette and M. Friedman (Eds.). Handbook of perception (Vol. 2). New York: Academic Press. 1974. (b)

Birnbaum. M. H., \& Veit, C. T. Seale-tree tests of an additive model for the size weight illusion. Perception \& Psychophysics. 1974. 16, 276-282.
Bogartz, R. S., \& Wackwitz. J. H. Polynomial response scaling and functional neasurement. Journal of Mathematical Psichologi. 1971, 8, $418-443$.

Curtis. D. W.. Attneave. F., \& Harrington. T. L. A test of a two-stage model of magnitude estimation. Perception \& Psychophysics, 1968. 3. 25.31.

Garner. W. R., Hake, H. W., \& Eriksen, C. W. Operationism and the concept of perception. Psychological Review. 1956. 63. 149-159.

Guilford. J. P. The perception of affective values. American Journal of Psychology. 1931. 43. 469-478.

Krantz. D. H.. Luce, R. D., Suppes, P.. \& Trersky, A. Foundations of measurement (Vol. 1). New York: Academic Press. 1971.

McBurney, D. H., Smith, D. V.. \& Shick, T. R. Gustatory cross-adaptation: Sourness and bitterness. Perception \& Psychophysics. 1972. 11. 228-232.

Moskowitz, H. R. Taste and food technology: Acceptability. aesthetics and preference. In E. C. Carterette and M. Friedman (Eds.). Handbook of perception (Vol. 6). New York: Academic Press. 1974.

SPENCE. W.. \& Guilford, J. P. The affective value of combinations of odors. American Joumal of Psychology. 1933. 45. $495-502$.

(Received for publication September 17, 1974: accepted April 25, 1975.) 